\title{
Development of a Method for Synthesizing Ag/Cu Composite Nanoparticles and Their Bonding Property
}

\author{
Takafumi Maeda ${ }^{1 *}$, Norihisa Matsubara ${ }^{1}$, Yoshio Kobayashi ${ }^{*}$, Yusuke Yasuda ${ }^{2}$, Toshiaki \\ Morita $^{2}$ \\ ${ }^{1}$ Department of Biomolecular Functional Engineering, College of Engineering, Ibaraki University, Hitachi, \\ Ibaraki 316-8511, Japan. \\ ${ }^{2}$ Hitachi Research Laboratory, Hitachi Ltd., Hitachi, Ibaraki 319-1292, Japan. \\ * Corresponding author. Tel.: +81-294-38-5052; email: yoshio.kobayashi.yk@vc.ibaraki.ac.jp \\ Manuscript submitted April 11, 2017; accepted August 8, 2017. \\ doi: 10.17706/ijmse.2017.5.3.102-109
}

\begin{abstract}
The present work describes synthesis of $\mathrm{Ag} / \mathrm{Cu}$ composite nanoparticles in aqueous solution, and metal-metal bonding in hydrogen gas or nitrogen gas using the nanoparticles. Synthesis of the $\mathrm{Ag} / \mathrm{Cu}$ composite nanoparticles was performed by the following process. First, a colloid solution of metallic $\mathrm{Cu}$ nanoparticles was prepared by reducing copper ions using hydrazine in an aqueous solution containing citric acid and polyvinylpyrrolidone. Then, metallic Ag nanoparticles were fabricated by reducing silver ions with hydrazine in the presence of the metallic $\mathrm{Cu}$ nanoparticles. The metallic Ag nanoparticles with a size of ca. 25 $\mathrm{nm}$ were immobilized on the metallic $\mathrm{Cu}$ nanoparticles with sizes of $60-120 \mathrm{~nm}$. $\mathrm{Cu}-\mathrm{Cu}$ bonding was performed by pressurizing metallic copper discs sandwiching the nanoparticles as a filler at $1.2 \mathrm{MPa}$ for 5 min under annealing in hydrogen gas or nitrogen gas. The shear strength, which was required to separate the bonded discs, recorded 26.8 and 12.1 MPa for the bonding in hydrogen gas and nitrogen gas, respectively.
\end{abstract}

Keywords: Metallic copper, metallic silver, nanoparticle, filler, meta-metal bonding.

\section{Introduction}

Currently in various industries such as automobile, civil engineering, construction industry and electronics industry, a metal-metal bonding is one of the most significant techniques. Pb-Sn solder, or filler has been used in various metal-metal bonding processes, because of its low melting point; the bonding desires low energy with no thermal damage to bonded sites.

The use of $\mathrm{Pb}$ has been limited, since $\mathrm{Pb}$ has toxicity property, which affects the environment as well as human health. Accordingly, development of Pb-free alloy fillers is quite a significant matter [1]-[4]. Recently, Sn-based alloys like $\mathrm{Sn}$-Cu have been paid attention for replacements of the Pb-based fillers, since the Sn-based alloys also have low melting points. If metal components like engines of vehicles that are manufactured by the bonding process using the Sn-based alloy filler are exposed to high temperature, thermal damage to the bonded metal components, or remelt of the filler between the bonded metals takes place, which releases the bonded metals [5]. Accordingly, though the fillers with low melting points can be used for low-temperature metal-metal bonding, they are not suitable for metal-metal bonding of materials exposed to high temperature.

Nanoparticles have special properties that differ from those of bulk materials as a consequence of their small size. Representative special properties are given as catalysis [6]-[8], magneticism [9], and melting 
point depression [10], [11]. The melting point depression property enables metallic nanoparticles to be used as fillers for metal-metal bonding performed at low temperature. The metal nanoparticles become bulk material after the bonding, so that the filler between the bonded metals does not remelt even for exposure to high temperature.

Metallic nanoparticles of Ag [12]-[15] and $\mathrm{Cu}$ [16]-[18] function as the filler. These nanoparticles have attracted extensive attention because of their high electrical conductivity. The Ag is chemically stable, promising that the Ag nanoparticle filler has a reliable bonding ability. However, concerns over its high cost and poor anti-migration property should be swept off. The $\mathrm{Cu}$ is also promising due to its lower cost and excellent anti-migration property. However, it tends to be oxidized in air, which provides unreliable bonding ability.

Kim et al. reported that the formation of $\mathrm{Ag} / \mathrm{Cu}$ composite nanoparticles prevented metallic $\mathrm{Cu}$ from being oxidized [19], which expects metallic $\mathrm{Cu}$ nanoparticles containing metallic Ag nanoparticles to have a reliable bonding ability. Yan et al. reported performance of metal-metal bonding with the use of $\mathrm{Ag} / \mathrm{Cu}$ composite nanoparticles [20]. Our research group has also studied on fabrication of $\mathrm{Ag} / \mathrm{Cu}$ composite nanoparticles and investigated their metal-metal bonding property [21], [22]. The process for fabricating the $\mathrm{Ag} / \mathrm{Cu}$ composite nanoparticles is complicated, since it is composed of fabrication of $\mathrm{Cu}$ oxide nanoparticles, reduction of the $\mathrm{Cu}$ oxide nanoparticles to metallic $\mathrm{Cu}$ nanoparticles, and reduction of $\mathrm{Ag}^{+}$in the presence of the $\mathrm{Cu}$ metal nanoparticles. For simplify the process, we proposed a method for producing $\mathrm{Ag} / \mathrm{Cu}$ composite nanoparticles [23]. The method was composed of only one step: The $\mathrm{Ag} / \mathrm{Cu}$ composite nanoparticles were fabricated by simultaneous reduction of $\mathrm{Ag}^{+}$and $\mathrm{Cu}^{2+}$ using hydrazine with polyvinylpyrrolidone (PVP) and citric acid as stabilizers. Their bonding abilities for performance of $\mathrm{Cu}-\mathrm{Cu}$ bonding in nitrogen gas or hydrogen gas were not very high: The formation of composite nanoparticles did not have a positive effect in the work. It is speculated that the Ag components were present not only on particle surface but also inside particles, and the presence of $\mathrm{Ag}$ components inside the metallic $\mathrm{Cu}$ nanoparticles prevented the metallic $\mathrm{Cu}$ nanoparticles from growing to metallic bulk, so that the simultaneous reduction method did not intensively work for bonding performance.

From this view point, the present work proposes an alternative method for fabricating the $\mathrm{Ag} / \mathrm{Cu}$ composite nanoparticles. The method is composed of reduction of the $\mathrm{Cu}^{2+}$ to produce metallic $\mathrm{Cu}$ nanoparticles, and reduction of $\mathrm{Ag}^{+}$to produce metallic $\mathrm{Ag}$ nanoparticles in the presence of the metallic $\mathrm{Cu}$ nanoparticles to immobilize the metallic Ag nanoparticles on the metallic $\mathrm{Cu}$ nanoparticle surface. The $\mathrm{Ag}$ nanoparticles are expected not to be inside the composite particles, which promotes the metallic $\mathrm{Cu}$ particle growth, and the following metal-metal bonding. Their metal-metal bonding property was also investigated.

\section{Experimental}

\subsection{Chemicals}

Starting chemicals used for producing the $\mathrm{Ag} / \mathrm{Cu}$ composite nanoparticles were copper nitrate trihydrate $\left(\mathrm{Cu}\left(\mathrm{NO}_{3}\right)_{2} \cdot 3 \mathrm{H}_{2} \mathrm{O}\right)\left(77.0 \%-80.0 \%\right.$ as $\left.\mathrm{Cu}\left(\mathrm{NO}_{3}\right)_{2}\right)$ and silver nitrate $\left(\mathrm{AgNO}_{3}\right)\left(99.8 \%\right.$ as $\left.\mathrm{AgNO}_{3}\right)$, respectively. Hydrazine monohydrate $(>98.0 \%)$ was used as a reducing reagent for metal ions. Citric acid monohydrate $(>99.5 \%)$ was used as an agent that chemically stabilizes $\mathrm{Cu}$ nanoparticles. PVP $(\mathrm{K}-30(\mathrm{Mw}=40000)$ ) was used as a dispersing agent in preparation of nanoparticles. All chemicals were purchased from Kanto Chemical Co., Inc., and were used as received. Water that was purified by ion-exchange with a distillation apparatus (Advantech RFD372NC) was used in all preparations.

\subsection{Preparation}

A method for preparing a colloid solution of $\mathrm{Ag} / \mathrm{Cu}$ composite nanoparticles is composed of two 
processes. One is production of precursor metallic $\mathrm{Cu}$ nanoparticles, and the other is production of metallic $\mathrm{Ag}$ nanoparticles in the presence of the metallic $\mathrm{Cu}$ nanoparticles. A colloid solution of metallic $\mathrm{Cu}$ nanoparticles was prepared by adding hydrazine to aqueous solution containing $\mathrm{Cu}\left(\mathrm{NO}_{3}\right)_{2}$, citric acid, and PVP with vigorous stirring at $40^{\circ} \mathrm{C}$. After $1 \mathrm{~h}$, a $\mathrm{AgNO}_{3}$ aqueous solution and hydrazine were added to the metallic $\mathrm{Cu}$ nanoparticle colloid solution to reduce $\mathrm{Ag}^{+}$ions in the presence of $\mathrm{Cu}$ nanoparticles. The reaction time was $2 \mathrm{~h}$. Initial concentrations of $\mathrm{Cu}, \mathrm{Ag}$, hydrazine, citric acid, and PVP were $0.0075 \mathrm{M}$, $0.0025 \mathrm{M}, 0.6 \mathrm{M}, 0.00025 \mathrm{M}$, and $1.0 \mathrm{~g} / \mathrm{L}$, respectively.

\subsection{Characterization}

The nanoparticles were characterized by transmission electron microscopy (TEM), X-ray diffractometry (XRD) and X-ray photoelectron spectroscopy (XPS). The TEM was performed with a JEOL JEM-2100 microscope operating at $200 \mathrm{kV}$. Samples for TEM were prepared by dropping and evaporating a particle colloid on a collodion-coated copper grid. The diameters of dozens of particles in the TEM images were measured to determine the number-averaged particle size and the standard deviation of the particle size distribution. The XRD data were collected using a Rigaku Ultima IV X-ray diffractometer equipped with a $\mathrm{CuK} \alpha 1$ radiation source operated at $40 \mathrm{kV}$ and $30 \mathrm{~mA}$. The powder samples for XRD measurement were prepared by removing the supernatant of the particle colloid solution using decantation and then drying the residue at room temperature for $24 \mathrm{~h}$ under vacuum. The surface composition of the particle was analyzed by the XPS using a JEOL JPS-9010 photoelectron spectrometer. The XPS spectra were recorded using $\mathrm{Mg}$-K $\alpha$ radiation ( $200 \mathrm{~W}, 10 \mathrm{kV}$, and $1253.6 \mathrm{eV}$ ). The XPS samples were the same as the XRD samples. To investigate the composition below the surface of the powder samples, the particles were etched using $500 \mathrm{kV} \mathrm{Ar}+$ ion sputtering.

The metal-metal bonding properties of the nanoparticles were investigated with a process composed of metal-metal bonding and measurement of bonding strength. The nanoparticle powder, which was obtained with the same process as that for the XRD sample, was sandwiched between a metallic copper stage disc with a diameter of $10 \mathrm{~mm}$ and a thickness of $5 \mathrm{~mm}$ and a metallic copper plate disc with a diameter of $5 \mathrm{~mm}$ and a thickness of $2.5 \mathrm{~mm}$. The discs sandwiching the powder were pressed at $1.2 \mathrm{MPa}$ while being annealed under nitrogen gas or hydrogen gas at $400^{\circ} \mathrm{C}$ for $5 \mathrm{~min}$ using a Shinko Seiki vacuum reflow system. For bonding strength measurement, the shear strength required to separate the bonded discs was measured with a Seishin SS-100KP bond tester.

\section{Results and Discussion}

\subsection{Morphology of Particles}

A TEM image of the Ag/Cu nanoparticles is shown in Fig 1. Smaller particles with a size of ca. $25 \mathrm{~nm}$ appeared to be immobilized on larger particles with sizes of 60-120 nm.

Fig. 2 shows an XRD pattern of the particles. Peaks were recorded at 43.3, 50.4 and 74.1 degree. These peaks were assigned to (111), (200) and (220) planes of cubic copper (JCPDS card No. 04-0836), respectively. Besides the peaks due to cubic copper, peaks were detected at 38.1, 64.4, and 77.5 degree, though some peaks were faint. They were assigned to the (111), (220), and (311) planes of cubic Ag metal (JCPDS card No. 04-0783), respectively. Though a peak due to (200) plane of cubic Ag should have appeared at 44.3 degree, the peak was not clearly detected because of overlap of the peak due to (200) plane of cubic Ag and the peak of (111) plane of cubic $\mathrm{Cu}$. These results indicated production of both Ag metal and $\mathrm{Cu}$ metal. Crystal sizes of the $\mathrm{Cu}$ and Ag metals were estimated on the basis of the (111) metallic $\mathrm{Cu}$ and $\mathrm{Ag}$ peaks, respectively, using the Scherrer equation. The crystal sizes of $\mathrm{Cu}$ metal and Ag metal was 31.7 and $10.8 \mathrm{~nm}$, respectively. 


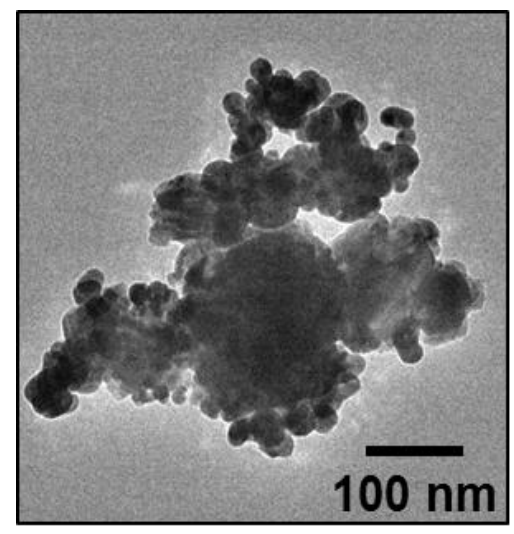

Fig. 1. TEM image of $\mathrm{Ag} / \mathrm{Cu}$ nanoparticles.

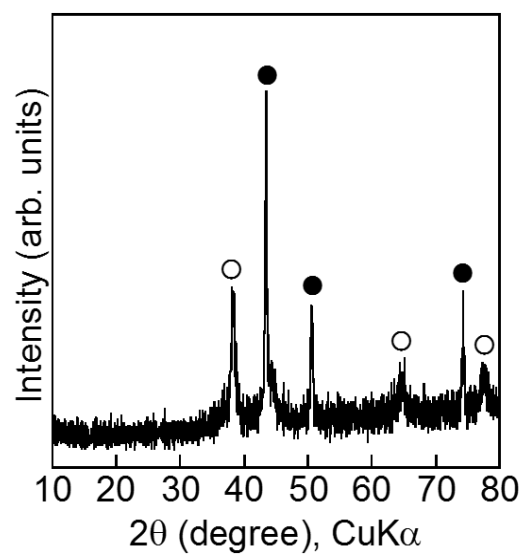

Fig. 2. XRD pattern of $\mathrm{Ag} / \mathrm{Cu}$ nanoparticles

$(\bullet)$ cubic $\mathrm{Cu},(\mathrm{O})$ cubic $\mathrm{Ag}$.

Fig. 3 shows the $\mathrm{Ag}+\mathrm{Cu}$ atomic ratios estimated from the XPS peak area intensity. For the as-prepared $\mathrm{Ag} / \mathrm{Cu}$ particles, the atomic ratios of $\mathrm{Cu}$ and $\mathrm{Ag}$ were 70 and 30 atom\%, respectively. The atomic ratios increased and decreased as the etching time increased, respectively. Those ratios reached 78 and 22 atom\%, respectively, at an etching number of 5. These results indicated that many species of Ag were present not inside the particles but on the particle surface. This indication implied that the large and small particles observed by the TEM (Fig. 1) were the metallic $\mathrm{Cu}$ and Ag nanoparticles, respectively, or the metallic Ag nanoparticles were immobilized on the metallic $\mathrm{Cu}$ nanoparticles.

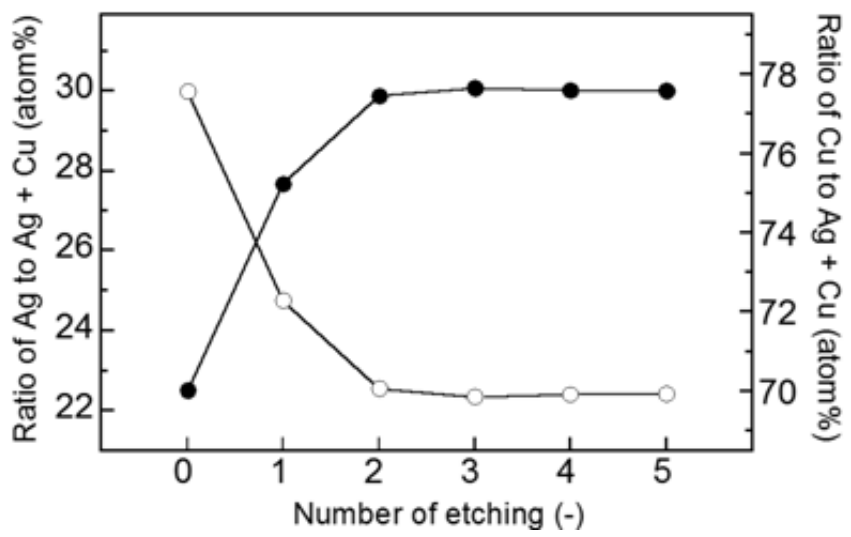

Fig. 3. Atomic ratios of $\mathrm{Ag}+\mathrm{Cu}$ for the surfaces of the $\mathrm{Ag} / \mathrm{Cu}$ nanoparticles as a function of the number of Ar$^{+}$etching steps. $(\bullet) \mathrm{Cu},(\circ) \mathrm{Ag}$.

\subsection{Bonding in Hydrogen Gas}

Bonding of the copper discs was performed in hydrogen gas for preventing the particles from being oxidized during bonding. Fig. 4 (a) shows a photograph of the copper stage disc after measurement of shear strength. Brown products that were obviously metallic $\mathrm{Cu}$ were observed in widespread area on the stage. This observation indicated that the nanoparticles were not oxidized, and still metallic $\mathrm{Cu}$. A shear strength was as high as $26.8 \mathrm{MPa}$ (Table 1). Since there is a mismatch in $d$-spacing between $\mathrm{Cu}$ and Ag metal, the presence of metallic Ag nanoparticles will deteriorate bonding of metallic $\mathrm{Cu}$ discs. However, the discs were strongly bonded using the $\mathrm{Ag} / \mathrm{Cu}$ nanoparticle powder. The Ag content in the particles of $25 \mathrm{~mol} \%$ was so low that the $\mathrm{Cu}-\mathrm{Cu}$ bonding was considered to be deteriorated. Fig. 4 (b) shows a SEM image of the disc surface after the measurement of shear strength. The nanoparticles appeared to be sintered. This observation of the particle sintering supported the strong bonding. 

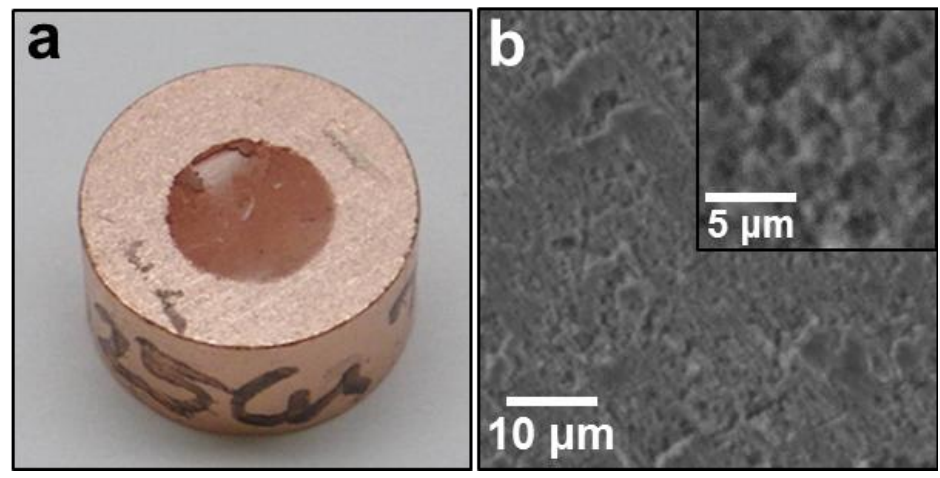

Fig. 4. Photograph (a) and SEM image (b) of metallic copper stage disc after measurement of shear strength. The bonding was carried out in hydrogen gas.

Table 1. Shear Strengths for Bonding in Different Atmospheres

\begin{tabular}{c|cc}
\hline Atmosphere & Hydrogen gas & Nitrogen gas \\
\hline Shear strength (Mpa) & 26.8 & 12.1 \\
\hline
\end{tabular}

\subsection{Bonding in Nitrogen Gas}

The previous section described that the copper discs were strongly bonded by performing the bonding in hydrogen gas. For safe performance of bonding in industry, the hydrogen gas was not desired to be used as atmosphere gas in the bonding. The present section describes the investigation on bonding with the use of the $\mathrm{Au} / \mathrm{Cu}$ nanoparticles in nitrogen gas. Fig. 5 (a) shows a photograph of the copper stage disc after measurement of shear strength. Brown products were also observed in widespread area on the stage. The brown color was darker than that for hydrogen gas, which implied that the nanoparticles were partially oxidized during the bonding because of the dark color derived from copper oxide. According to our previous works on metallic $\mathrm{Cu}$ nanoparticles [24],[25], a slight amount of copper oxide is present in the particles, though the main component is metallic $\mathrm{Cu}$. The copper oxide might have been slightly present on the metallic $\mathrm{Cu}$ surface of the $\mathrm{Ag} / \mathrm{Cu}$ nanoparticles, and the copper oxide grew and revealed the dark color due to copper oxide, which implied that the copper discs were not strongly bonded compared to in hydrogen gas. Fig. 5 (b) shows a SEM image of the disc surface after the measurement of shear strength. Several particles were observed with no sintering. This observation also implied the non-strong bonding. A shear strength was 12.1 MPa (Table 1), which was lower than that of in hydrogen gas. The lower shear strength was predicted by the implications obtained by the photograph and the SEM image. Probably, the partial oxidation of the particle surface deteriorated the bonding. In our previous work on $\mathrm{Ag} / \mathrm{Cu}$ nanoparticles fabricated by simultaneous reduction of $\mathrm{Cu}^{2+}$ and $\mathrm{Ag}^{+}$in aqueous solution [23], the nanoparticle powder did not intensively help bonding of copper discs in nitrogen gas. This meant that the $\mathrm{Ag} / \mathrm{Cu}$ nanoparticles produced in the present work was superior to those in the previous work for bonding in nitrogen gas. The metallic $\mathrm{Ag}$ and $\mathrm{Cu}$ components were expected to be dispersed in the particles for the simultaneous reduction, because metallic $\mathrm{Ag}$ and metallic $\mathrm{Cu}$ were simultaneously produced. In the present work, the metallic Ag was produced in the presence of the metallic $\mathrm{Cu}$ nanoparticles. As a result, the metallic $\mathrm{Ag}$ component was not dispersed inside the $\mathrm{Ag} / \mathrm{Cu}$ particles but immobilized on the metallic $\mathrm{Cu}$ particle surface, as supported by the TEM observation and the XPS measurement. The metallic Ag component on surface was considered to cover copper oxide on particle surface and control the growth of copper oxide in an early stage of bonding. Consequently, the discs were strongly bonded even in nitrogen gas. 


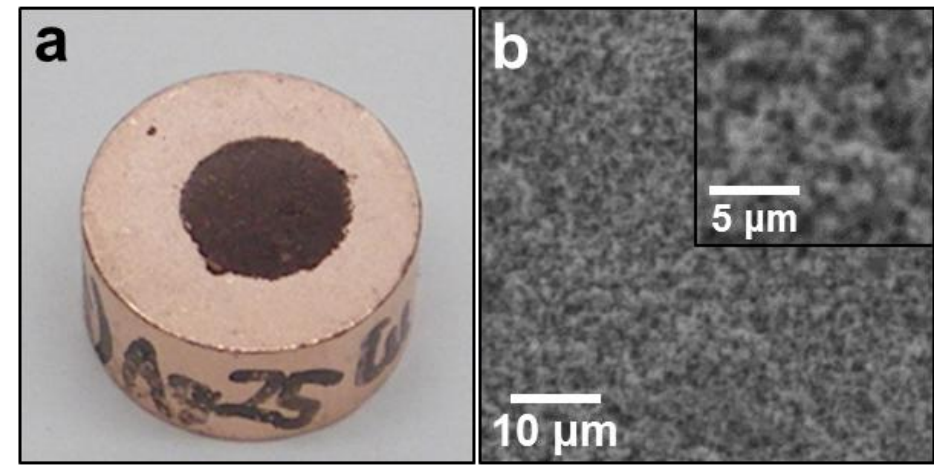

Fig. 5. Photograph (a) and SEM image (b) of metallic copper stage disc after measurement of shear strength. The bonding was carried out in nitrogen gas.

\section{Conclusion}

The $\mathrm{Ag} / \mathrm{Cu}$ composite nanoparticles, in which the metallic $\mathrm{Ag}$ nanoparticles with the size of ca. $25 \mathrm{~nm}$ were immobilized on the metallic Cu nanoparticles with the sizes of $60-120 \mathrm{~nm}$, were fabricated with the method composed of two processes. The first step was the fabrication of metallic $\mathrm{Cu}$ nanoparticles by the reduction of copper ions in the aqueous solution, and the second was the fabrication of metallic Ag nanoparticles by the reduction of silver ions in the aqueous solution containing the metallic $\mathrm{Cu}$ nanoparticles. The shear strengths required to separate metallic copper discs bonded in hydrogen gas and nitrogen gas at $400^{\circ} \mathrm{C}$ for 5 min while pressed at $1.2 \mathrm{MPa}$ were as high as 26.8 and $12.1 \mathrm{MPa}$, respectively. The strong bonding was performed even in nitrogen, because the metallic Ag on particle surface covered copper oxide on metallic $\mathrm{Cu}$ surface, which helped the $\mathrm{Cu}-\mathrm{Cu}$ bonding process.

\section{Acknowledgment}

This work was partially supported by the Hitachi Ltd.

\section{References}

[1] Zhao, X., Wen, Y., Li, Y., Liu, Y., \& Wang, Y. (2016). Effect of $\gamma-\mathrm{Fe}_{2} \mathrm{O}_{3}$ nanoparticles size on the properties of Sn-1.0Ag-0.5Cu nano-composite solders and joints. Journal of Alloys and Compounds, 662, 272-282.

[2] Lee, S. H., Yoo, K., Jha, M. K., \& Lee, J. C. (2015). Separation of Sn from waste Pb-free Sn-Ag-Cu solder in hydrochloric acid solution with ferric chloride. Hydrometallurgy, 157, 184-187.

[3] Sundelin, J. J., Nurmi, S. T., Lepistö, T. K., \& Ristolainen, E. O. (2006). Mechanical and microstructural properties of SnAgCu solder joints. Materials Science and Engineering: A, 420(1-2), 55-62.

[4] Koo, J., Lee, C., Hong, S. J., Kim, K. S., \& Lee, H. M. (2015). Microstructural discovery of Al on Sn-0.5Cu-based Pb-free solder design. Journal of Alloys and Compounds, 650, 106-115.

[5] Johnson, R. W., Evans, J. L., Jacobsen, P., Thomson, J. R., \& Christopher. M. (2004). The changing automotive environment: High-temperature electronics. IEEE Transactions on Electronics Packaging Manufacturing, 27(3), 164-176.

[6] Baeza, J. A., Calvo, L., Rodriguez, J. J., \& Gilarranz, M. A. (2016). Catalysts based on large size-controlled Pd nanoparticles for aqueous-phase hydrodechlorination. Chemical Engineering Journal, 294, 40-48.

[7] Wu, G., Liang, X., Zhang, H., Zhang, L., Yue, F., Wang, J., et al. (2016). Highly stable and sub-3 nm Ni nanoparticles coated with carbon nanosheets as a highly active heterogeneous hydrogenation catalyst. Catalysis Communications, 79, 63-67.

[8] Park, J., Kim, S., Sim, Y., Yoon, O. J., Han, M. S., Yang, H. S., et al. (2016). Simple synthesis of high-quality 
CdS nanowires using Au nanoparticles as catalyst. Journal of Alloys and Compounds, 659, 38-43.

[9] Li, O. A., Lin, C. R., Chen, H. Y., Hsu, H. S., Shih, K. Y., Edelman, I. S., et al. (2016). Size dependent magnetic and magneto-optical properties of $\mathrm{Ni}_{0.2} \mathrm{Zn}_{0.8} \mathrm{Fe}_{2} \mathrm{O}_{4}$ nanoparticles. Journal of Magnetism and Magnetic Materials, 408, 206-212.

[10] Luo, W., \& Hu, W. (2013). Gibbs free energy, surface stress and melting point of nanoparticle. Physica B: Condensed Matter, 425, 90-94.

[11] Zolriasatein, A., \& Shokuhfar, A. (2015). Size effect on the melting temperature depression of $\mathrm{Al}_{12} \mathrm{Mg}_{17}$ complex metallic alloy nanoparticles prepared by planetary ball milling. Physica E: Low-dimensional Systems and Nanostructures, 74, 101-107.

[12] Akada, Y., Tatsumi, H., Yamaguchi, T., Hirose, A., Morita, T., \& Ide, E. (2008). Interfacial bonding mechanism using silver metallo-organic nanoparticles to bulk metals and observation of sintering behavior. Materials Transactions, 49(7), 1537-1545.

[13] Yasuda, Y., Ide, E., \& Morita, T. (2009). Low-temperature bonding using silver nanoparticles stabilized by short-chain alkylamines. Japanese Journal of Applied Physics, 48(12R), 125004-1-125004-5.

[14] Alarifi, H., Hu, A., Yavuz, M., \& Zhou, Y. N. (2011). Silver nanoparticle paste for low-temperature bonding of copper. Journal of Electronic Materials, 40(6), 1394-1402.

[15] Guo, W., Zeng, Z., Zhang, X., Peng, P., \& Tan, S. (2015). Low-temperature sintering bonding using silver nanoparticle paste for electronics packaging. Journal of Nanomaterials, 2015, 897142-1-897142-7.

[16] Hiroshi, N., Tomoaki, H., \& Tadashi, T. (2011). Bonding process of $\mathrm{Cu} / \mathrm{Cu}$ joint using $\mathrm{Cu}$ nanoparticle paste. Transactions of JWRI, 40(2), 33-36.

[17] Maeda, T., Kobayashi, Y., Yasuda, Y., \& Morita, T. (2014). Metal-metal bonding properties of copper oxide nanoparticles. e-Journal of Surface Science and Nanotechnology, 12, 105-108.

[18] Kobayashi, Y., Abe, Y., Maeda, T., Yasuda, Y., \& Morita, T. (2014). A metal-metal bonding process using metallic copper nanoparticles produced by reduction of copper oxide nanoparticles. Journal of Materials Research and Technology, 3(2), 114-121.

[19] Kim, C. K., Lee, G. J., Lee, M. K., \& Rhee, C. K. (2014). A novel method to prepare Cu@Ag core-shell nanoparticles for printed flexible electronics. Powder Technology, 263, 1-6.

[20] Yan, J., Zou, G., Zhang, Y., Li, J., Liu, L., Wu, A., et al. (2013). Metal-metal bonding process using Cu+Ag mixed nanoparticles. Materials. Transactions, 54(6), 879-883.

[21] Kobayashi, Y., Shirochi, T., Yasuda, Y., \& Morita, T. (2013). Synthesis of silver/copper nanoparticles and their metal-metal bonding property. Journal of Mining and Metallurgy, Section B: Metallurgy, 49, 65-70.

[22] Kobayashi, Y., Maeda, T., Yasuda, Y., \& Morita, T. (2016). Metal-metal bonding using silver/copper nanoparticles. Applied Nanoscience, 6, 883-893.

[23] Maeda, T., Matsubara, N., Kobayashi, Y., Yusuke, Y., \& Morita, T. Fabrication of Ag/Cu composite nanoparticles for metal-metal bonding. Materials Science and Technology, submitted.

[24] Kobayashi, Y., Shirochi, T., Yasuda, Y., \& Morita, T. (2011). Preparation of metallic copper nanoparticles in aqueous solution and their bonding properties. Solid State Sciences, 13(3), 553-558.

[25] Kobayashi, Y., Shirochi, T., Yasuda, Y., \& Morita, T. (2012). Metal-metal bonding process using metallic copper nanoparticles prepared in aqueous solution. International Journal of Adhesion \& Adhesives, 33, 50-55. 


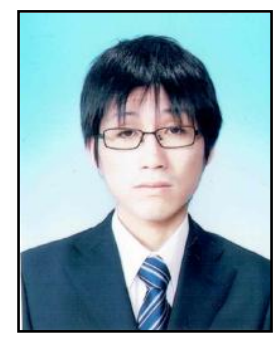

Takafumi Maeda graduated from the College of Engineering, Ibaraki University in 2010. He got his Ph.D. in the Graduate School of Science and Engineering, Ibaraki University in 2015. He worked at Kitami Institute of Technology as an assistant professor (2015-2016).

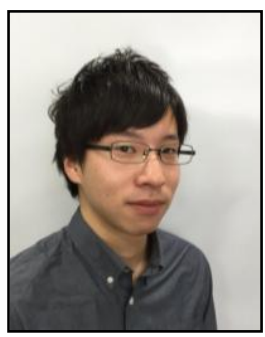

Norihisa Matsubara got the bachelor's degree at the College of Engineering, Ibaraki University in 2015. He has been a student in the Graduate School of Engineering, Nagoya Institute of Technology since 2015.

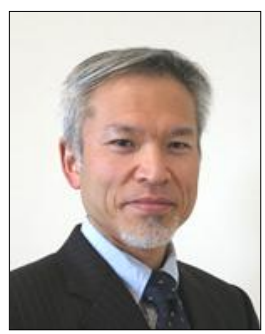

Yoshio Kobayashi received his B.S. (1988), M.S. (1990), and Ph.D. (1993) degrees from Tohoku University. He joined the Institute for Chemical Reaction Science, Tohoku University, as a research associate (1993-1997). He worked at the Department of Chemistry, Colorado State University (1997-1998) and the Department of Chemistry, Kansas State University (1998-1999), as postdoctoral researcher. He moved to the Departamento de Química Física, Universidade de Vigo, Spain (1999-2001) as a visiting researcher invited by the Ministry of Education of the Spanish government. He joined the Department of Chemical Engineering, Graduate School of Engineering, Tohoku University in 2001 as a research associate and in 2003 as an associate professor. He has been employed in the College of Engineering, Ibaraki University since 2006 as a professor.

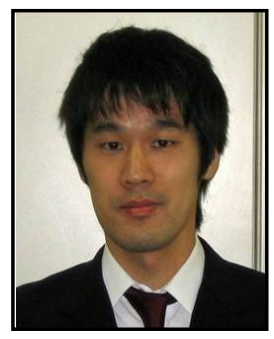

Yusuke Yasuda graduated from the Faculty of Engineering, Tokyo Institute of Technology in 2005. He got his Ph.D. in the Graduate School of Engineering, Tokyo Institute of Technology in 2012. He has been employed in Hitachi Research Laboratory, Hitachi Ltd. since 2005.

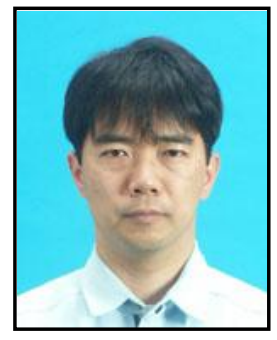

Toshiaki Morita graduated from the Faculty of Engineering, Kyusyu University in 1991. He got his Ph.D. in the Graduate School of Engineering, Osaka University in 2008. He has been employed in Hitachi Research Laboratory, Hitachi Ltd. since 1993. 